\title{
Organelles Stress and their Crosstalk within Diabetic Myocardium
}

\author{
By Doina Popov ${ }^{*}$
}

Diabetes-associated cardiovascular dysfunction is characterized by homeostasis perturbation induced by systemic stressors, such as hyperglycemia, excess of reactive oxygen and nitrogen species, shear-stress, and inflammatory environment. The recent data highlight the aggravating effect of local, organelles-related stress, manifest in mitochondria, endoplasmic reticulum (ER), lysosomes, proteasomes, inflammasomes. The occurrence of local stress might allow its alleviation inside the cell, at organelles level, a novel strategy potentially more efficient compared to current systemic therapeutic approaches. The aim of this article is to link the electron microscopy evidence on mitochondrial stress in diabetic coronary endothelium and cardiomyocytes (CMs) to the newly identified molecules/ mechanisms beyond it. The issues examined are: (i) Mitochondrial homeostasis and "quality control" mechanisms within myocardium, (ii) Mitochondrial dysfunction and dynamics within diabetic myocardium: fusion, fission, and mitophagy events, (iii) The molecular crosstalk between mitochondria, ER, lipid droplets, and cytoplasm, (iv) Perspectives. At the horizon, targeting mitochondrial dynamics mediators, deciphering the defects in mitochondrial cell signaling control, understanding mitochondria retrograde signaling, and manipulation of ER stress-associated lipid droplets formation may conduct to novel drugs aimed to preserve CMs viability and to alleviate diabetesinduced heart damage.

Keywords: Diabetic myocardium, mitochondrial stress, organelles-related stress

\section{Introduction}

The interest of scientists returned to mitochondria, once novel properties of these organelles have been recently unveiled. Thus, mitochondria exert "quality control" mechanisms (via fusion, fission, and mitophagy), translocate to the subcellular territories where an energy supply is needed, and exert signaling functions by regulation of local reactive oxygen species (ROS) concentrations (Al-Mehdi et al. 2012). These recent areas are receiving much of attention, as understanding of molecular mechanisms beyond them may explain the survival/death of cells in pathological conditions. The knowledge on endothelial cells (ECs) and cardiomyocytes (CMs) survival within diabetic myocardium is related to installment of mitochondrial dysfunction, an event more relevant to disease than previously thought. In particular, deciphering mitochondrial turnover regulation and the role of mitochondrial dynamics in diabetic cardiomyopathy may potentially lead to innovative therapeutic

${ }^{*}$ Head of the Pathophysiology and Pharmacology Department, Institute of Cellular Biology and Pathology "N. Simionescu" of the Romanian Academy, Romania. 
strategies aiming cellular survival and disease control (Barazzoni 2012, Dai et al. 2012, Wang et al. 2012). Here, we link the electron microscopy evidence on mitochondrial dysfunction in diabetic coronary endothelium and CMs (original images obtained in our laboratory) to the newly identified molecules/mechanisms beyond it. The review is organized into the following sections: (i) Mitochondrial homeostasis and "quality control" mechanisms within myocardium, (ii) Mitochondrial dysfunction and dynamics within diabetic myocardium: fusion, fission, and mitophagy events, (iii) The molecular crosstalk between mitochondria, endoplasmic/sarcoplasmic reticulum (ER/SR), lipid droplets, and cytoplasm and (iv) Perspectives.

\section{Mitochondrial Homeostasis and Quality Control Mechanisms Within Myocardium}

Adaptation of mitochondria content according to the specific cellular energy requirement (ATP generation) that sustains physiological growth is identified as mitochondrial homoeostasis (Groenewoud and Zwartkruis 2013). The latter process is achieved by several quality control mechanisms, such as the repair of lipids, proteins, and mitochondrial DNA (mtDNA) damages, the regulation of mitochondria amount and functionality with preservation of the healthy organelles and elimination of the dysfunctional ones (by mitophagy, a specialized form of autophagy), and the fine-tuning of mitochondriogenesis (Groenewoud and Zwartkruis 2013, Palikaras and Tavernarakis 2014). Within heart microvasculature and myocytes, mitochondrial homeostasis is tightly regulated, as this organelle houses both the electron transport chain (source of oxidative stress) and the antioxidant defense enzymes (e.g. superoxide dismutase, glutathione peroxidase).

In vascular ECs, the mitochondrial content is rather modest and the energy demand is relatively low, consistent to cells quiescent phenotype in a physiological context. Nevertheless, mitochondria form networks and exert quality control mechanisms, ensuing the normal angiogenic and vasodilator function of ECs (Kluge et al. 2013). The mechanism beyond physiologic angiogenesis involves upregulation of endogenous Uncoupling Protein 2 (UCP2) that decreases the mitochondrial membrane potential $(\Delta \psi)$ produced by the electron transport chain, and limits superoxide anions generation (Shimasaki et al. 2013). As for vasodilation, this may be induced by $\mathrm{H}_{2} \mathrm{O}_{2}$ released by mitochondria in a setting of flow exposure of human coronary capillaries (Bubolz et al. 2012). ECs mitochondria activate also signaling mechanisms, and generated ROS are important for physiological responses to nutrient status, hypoxia, and shear stress (Kluge et al. 2013).

Compared to ECs, CMs are rich in mitochondria (occupying one-third of the cell volume), consistent with the high energy demand of the heart; it is estimated that $90 \%$ of the energy consumed by myocardial contraction is provided by mitochondrial oxidative phosphorylation (Marzetti et al. 2013, Schaper et al. 1985). Moreover, within CMs the turnover of these organelles is 
strictly controlled, in order to preserve the normal energy production by "healthy" mitochondria; the main pathways of mitochondrial turnover are mitophagy, proteases, proteasome, and release of mitochondrial-derived vesicles (MDV). Mitophagy clears the malfunctioning mitochondria via their engulfment in autophagosomes, that fuse with lysosomes allowing hydrolytic degradation; the protease-proteasome pathways assist elimination of damaged mitochondria, while MDV sort and transport oxidized proteins and lipids directly to the lysosomes, ensuing removal of damaged organelles content; the latter pathway operates as a mitophagy-independent mitochondrial quality control mechanism (Dai et al. 2012, Galluzzi et al. 2012, McBride and Scorrano 2013, McLelland et al. 2014, Shutt and McBride 2013). Interestingly, the mammalian CMs contain three subpopulations of mitochondria, with distinct intracellular location, morphology, biochemical properties, and targets of the generated ATP (Hollander et al. 2014). These are the sub-sarcolemmal mitochondria (SSM), interfibrilar mitochondria (IFM) and perinuclear mitochondria (Pn) (Table 1). SSM are positioned beneath the sarcolemma (Figure 1a) and are influenced by insulin resistance and heart ischemia (Davidson 2011). IFM are packed between myofibrils and have a relatively fixed location imposed by the physical constraint of the sarcomeres (Figure 1b). Recent evidence prove that IFM are subjected to dynamic shape changes by either fusion into elongated organelles or fragmentation (fission) into smaller units (Figures $2 \mathrm{~b}$ and $2 \mathrm{~d}$ ). The perinuclear mitochondria are located at nuclear poles, in the sarcomere-free region (Figure 1c) (Baseler et al. 2011, Davidson 2011, Hollander et al. 2014).

Figure 1. Heterogeneity of Cardiomyocyte Mitochondria as demonstrated by Electron Microscopy. (a) Sub-sarcolemmal Mitochondria (SSM), (b) Interfibrilar Mitochondria (IFM), (c) Perinuclear Mitochondria (Pn)

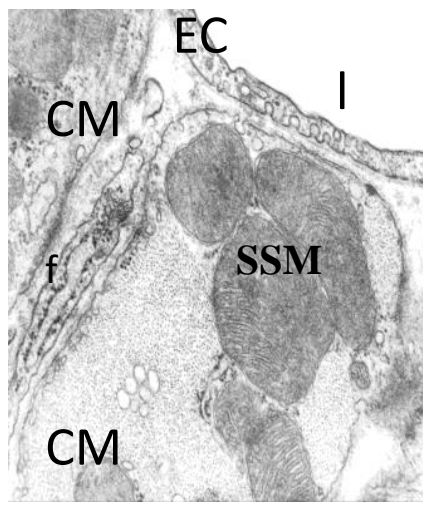

a

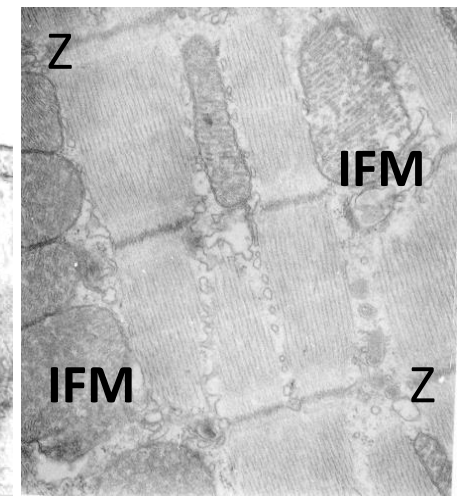

b

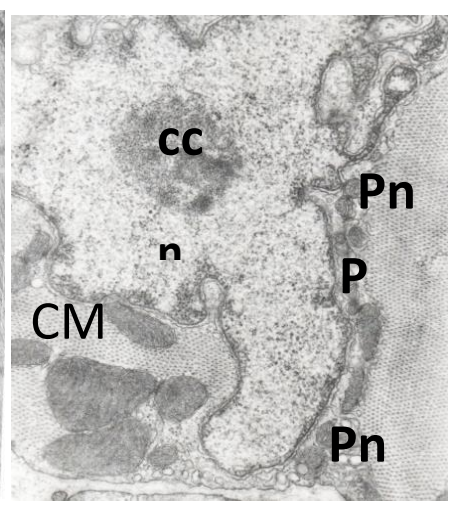

C

Abbreviations: EC, endothelial cell; CM: cardiomyocyte; 1: capillary lumen; f: fibroblast; Z: Z band; n: nucleus; cc: condensed chromatin.

Original magnifications: (a) x54,600; (b) and (c) x36,450 
Table 1. Heterogeneity of Cardiomyocyte Mitochondria: The Specific Properties According to Intracellular Location

\begin{tabular}{|c|c|c|c|c|}
\hline $\begin{array}{l}\text { Spatial location within } \\
\text { the cardiomyocyte }\end{array}$ & $\begin{array}{l}\text { Sub-sarcolemmal } \\
\text { mitochondria }\end{array}$ & Interfibrilar mitochondria & Perinuclear & References \\
\hline Size & No special feature & $\begin{array}{l}\text { Elongated/ } \\
\text { fragmented }\end{array}$ & $\begin{array}{l}\text { Significantly smaller, round, } \\
\text { with frequent nanotubules } \\
\text { suggestive for content } \\
\text { transfer }\end{array}$ & $\begin{array}{c}\text { Marín-García } 2013 \\
\text { Huang et al. } 2013\end{array}$ \\
\hline Cristae morphology & Lamelliform & Tubular & $\begin{array}{l}\text { Poorly developed } \\
\text { (in immature CM) }\end{array}$ & Davidson 2011 \\
\hline Biochemical properties & $\begin{array}{l}\text { Contains } \mathrm{Cx} 43 \text {, responsive to } \\
\text { FGF-2, higher }\left[\mathrm{Ca}^{2+}\right] \text { mito levels }\end{array}$ & $\begin{array}{l}\text { Cx43 negative, less responsive } \\
\text { to FGF-2,lower }\left[\mathrm{Ca}^{2+}\right] \text { mito } \\
\text { levels, posttranslational } \\
\text { modifications (oxidations and } \\
\text { deamidations), decreased } \\
\text { abundance of proteins of fatty } \\
\text { acids oxidation and electron } \\
\text { transport chain pathways }\end{array}$ & $\begin{array}{l}\text { Form a dynamic perinuclear } \\
\text { network involved in } \\
\text { mitochondria -to -nucleus } \\
\text { signal transduction and } \\
\text { coordination }\end{array}$ & $\begin{array}{l}\text { Srisakuldee et al. } 2014 \\
\text { Holmuhamedov et al. } \\
2012 \\
\text { Baseler et al. } 2011 \\
\text { Huang et al. } 2013\end{array}$ \\
\hline $\begin{array}{l}\text { Target of synthesized } \\
\text { ATP }\end{array}$ & $\begin{array}{l}\text { Plasma membrane ion pumps, } \\
\text { maintenance of the plasma } \\
\text { membrane potential and internal } \\
\text { stores of calcium, generation of } \\
\text { the cardiac action potential }\end{array}$ & $\begin{array}{l}\text { CMs contraction, efficient } \\
\text { cardiac energy utilization }\end{array}$ & Transcription and translation & Davidson 2011 \\
\hline Response to $\mathrm{H}_{2} \mathrm{O}_{2}$ & Similar to IFM & Similar to SSM & & Zhang and Shah 2014 \\
\hline $\begin{array}{l}\text { Response to superoxide } \\
\text { production and mPTP } \\
\text { transition }\end{array}$ & Preferential & $\begin{array}{l}\text { Generate more superoxide, } \\
\text { susceptible to mPTP opening }\end{array}$ & & $\begin{array}{l}\text { Baseler et al. } 2011 \\
\text { Zhang and Shah } 2014\end{array}$ \\
\hline Response to heart failure & $\begin{array}{l}\text { No effect on pyruvate } \\
\text { dehydrogenase turnover, modest } \\
\text { effect on respiratory function }\end{array}$ & $\begin{array}{l}\text { Decreased pyruvate } \\
\text { dehydrogenase turnover and } \\
\text { maximal rate of mitochondrial } \\
\text { respiration }\end{array}$ & & Shekar et al. 2014 \\
\hline
\end{tabular}




\begin{tabular}{|l|l|l|l|l|}
\hline $\begin{array}{l}\text { Response to ischemic } \\
\text { insult }\end{array}$ & More vulnerable & Less vulnerable & $\begin{array}{l}\text { Holmuhamedov et al. } \\
2012\end{array}$ \\
\hline Cardio-protection in IR & Diazoxide is effective & Diazoxide is less effective & $\begin{array}{l}\text { Holmuhamedov et al. } \\
2012\end{array}$ \\
\hline Response to diabetes & & $\begin{array}{l}\text { Sensitive, cristae express } \\
\text { Mitofilin and mtHsp70 }\end{array}$ & $\begin{array}{l}\text { Baseler et al. 2011 } \\
\text { Davidson 2011 }\end{array}$ \\
\hline
\end{tabular}

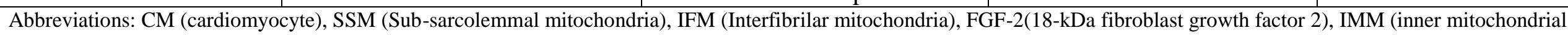
membrane), mPTP (mitochondrial permeability transition pore), mtHsp70 (mitochondrial heat shock protein 70), IR (ischemia-reperfusion) 
CMs mitochondria form dynamic membrane networks and may undergo intracellular redistributions according to the metabolic demands and the quality control mechanisms, e.g. organelles translocation to control local ROS concentrations (Al-Mehdi et al. 2012). The control mechanism may be either acute (conducting to the qualitative modification of mitochondrial function) or long-term transcriptional (increasing mitochondrial number) (Gao et al. 2014). Additionally, in areas of high mitochondrial density (such as in Figure 3b), the molecules released by a single mitochondrion may influence the function of their local neighbors (Eisner et al. 2013). Within myocardium, mitochondria homeostasis is perturbed by excessive oxidative stress and $\mathrm{Ca}^{2+}$ accumulation. Both disturbing factors are located in the inner mitochondrial membrane (IMM): the incomplete reduction of molecular oxygen during electron transport generates ROS, while the $\mathrm{Ca}^{2+}$-dependent opening of mPTP leads to the diminishment of $\Delta \psi$, interruption of ATP generation, and CMs death.

\section{Mitochondrial Dysfunction and Dynamics Within Diabetic Myocardium: Fusion, Fission and Mitophagy Events}

The imbalance in homeostasis along with dysregulation of mitochondrial turnover portray mitochondrial dysfunction (Dai et al. 2012) . Considered a "sine qua non of cardiovascular disease", mitochondrial dysfunction is implicated in cardiac hypertrophy, myocardial infarction, ischemia-reperfusion injury (IRI), arrhythmias, atherosclerosis, and hypertension (Hill 2013). Moreover, ischemic heart disease and cardiomyopathy have been linked to accumulation of mitochondrial DNA mutations (Dominic et al. 2014). Dysfunctional mitochondria are less bioenergetically efficient, producing reduced ATP levels for heart contraction, and excessive ROS levels (Marzetti et al. 2013). The causal role of mitochondrial dysfunction in heart pathology (likewise in cancer and neurodegeneration) attracted numerous novel studies, highlighting the molecular mechanisms involved. Today, it is established that three active damages control mechanisms operate beyond mitochondrial dysfunction: (i) the dynamic morphology/shape changes, via continuous cycles of fusion and fission (Anand et al. 2014), (ii) the defective clearance of malfunctioning mitochondria by mitophagy and (iii) the inadequate replenishment of the cellular mitochondrial pool by biogenesis (Groenewoud and Zwartkruis 2013, Marzetti et al. 2013, Palikaras and Tavernarakis 2014). Taken together, mitochondrial dynamics events contributes to preservation of mitochondria function inside the cells (tilts the imbalanced ATP generation towards an increase in energy efficiency), prevents loss of metabolic substrates or of mtDNA, and regulates cardiac homeostasis and adaptation to stress (Ikeda et al. 2014). The fusion of outer mitochondrial membrane (OMM) and of IMM in neighboring mitochondria allows mixing and uniform distribution of their internal compartments (such as soluble matrix and intermembrane space constituents) (Eisner et al. 2013, Gao et al. 2014). The common situation is the fusion of dysfunctional mitochondria with "healthy" ones, transiently buffering 
the defects and ensuing increased metabolic and bioenergetics efficacy (Lackner 2014, Youle and van der Bliek 2012). Recently, other biological advantages of this process have been disclosed, such as resistance to mitophagy and apoptosis (as the large size mitochondria are difficult to be engulfed by autophagosomes), enhancement of energy supply, and limitation of agedependent mtDNA mutations (Escobar-Henriques and Anton 2013, Ikeda et al. 2014, Shutt and McBride 2013). Under stress conditions, fusion is beneficial for the heart (Ikeda et al. 2014). Initiation of mitochondrial fusion requires activation of fusion molecules and inhibition of mitochondrial fragmentation/fission route (Shutt and McBride 2013). The fusion molecules are transmembrane GTPases belonging to the dynamin family: mitofusin 1 (MFN1) and mitofusin 2 (MFN2) regulate OMM fusion, while optic atrophy protein 1 (OPA1) intervenes in IMM fusion (Baker et al. 2014). Within diabetic myocardium, dysfunctional capillary ECs display frequent fusion, i.e. the interconnected organelles generate tubular-shaped elongated mitochondria that occupy almost the whole cell (Figure 2a). In diabetic CMs, fusion of IFM conduct to assembly of organelles even longer than a sarcomere (Figure 2b). Reports indicate that membrane fusing activity of MFN-1 and 2 creates holes on the OMM and facilitates the stress-induced mitochondrial permeability transition process with substantial consequences on myocyte survival (Papanicolaou et al. 2012). However, mitochondrial "hyperfusion" appears as protective during the early response to cellular stress, but detrimental at long term, because accumulated dysfunctional mitochondria (source of ROS overproduction and of cell death inducers) may damage the "healthy" ones (Shutt and McBride 2013). In the latter circumstance, fusion is inhibited by the short isoform of OPA1, generated by stress-induced OPA1 proteolytic processing (Anand et al. 2014). 
Figure 2. Mitochondria Fusion and Fission in Myocardial Capillaries ( $a$ and $c$ ) and Cardiomyocytes ( $b$ and $d$ ) as Shown by Electron Microscopy

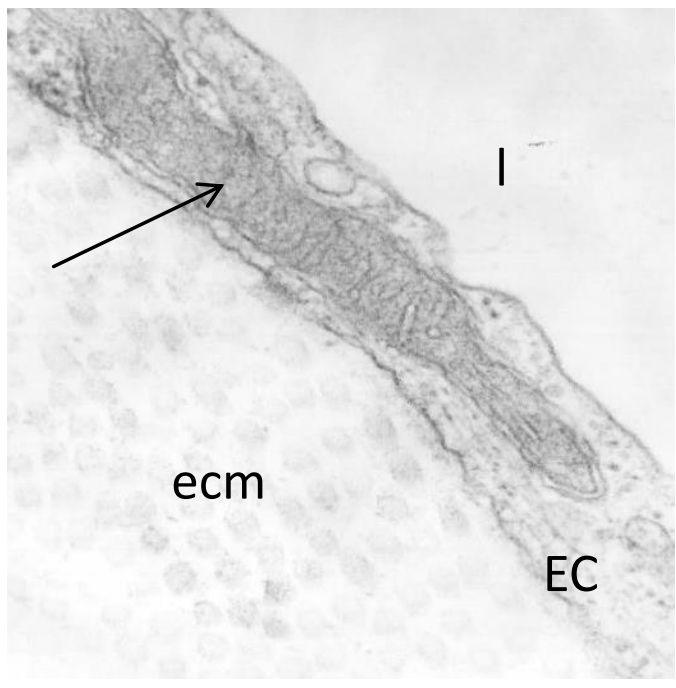

a

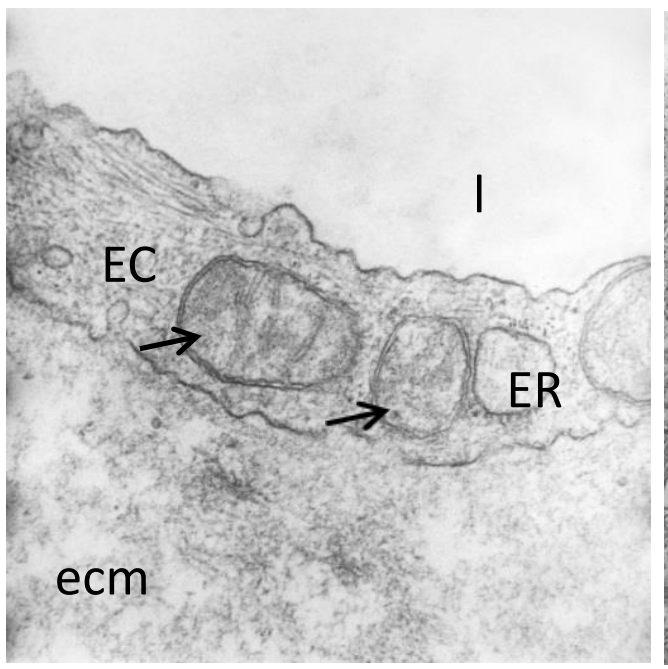

C

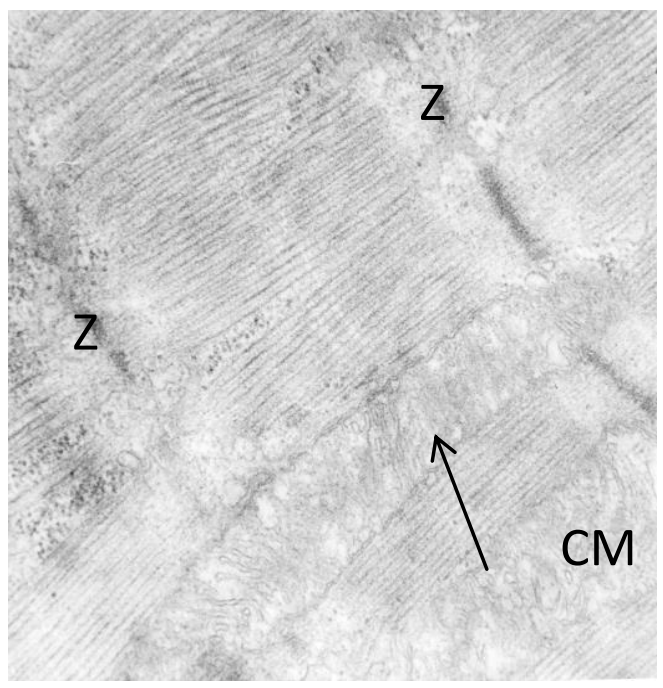

b

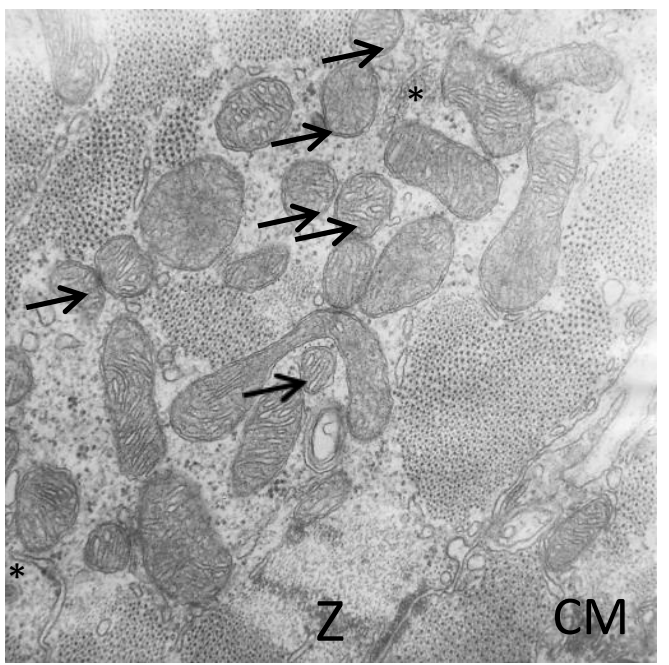

d

Abbreviations: EC, endothelial cell; CM: cardiomyocyte; 1: capillary lumen; Z: Z band; ecm: extracellular matrix; long arrows: fused mitochondria; short arrows: fragmented mitochondria indicative of fission; ER: endoplasmic reticulum; *: potential contact points with ER/SR cisternae, where fission is initiated.

Original magnifications: (a) and (c) x83,400; (b) and (d) x54,600.

The dynamic shape changes of mitochondria are interrelated, as the elongated structures are subjected to fission resulting in formation of two distinct populations of smaller mitochondria: (i) the dysfunctional ones, committed to clearing by mitophagy and (ii) the "healthy" organelles, assigned for further fusions. The tags for recognition of dysfunctional mitochondria are depolarization (associated with reduced $\Delta \psi$ ) and presence of Parkin (with ubiquitin ligase activity) and of PTEN-induced kinase 1 (PINK1); the "healthy" mitochondria display normal $\Delta \psi$ and components (Gao et al. 2014, Ikeda et al. 
2014, Jin and Youle 2012, Shlevkov and Schwarz 2014, Shutt and McBride 2013). Additionally to the fused, elongated mitochondria, the normal size organelles may be a source for dysfunctional small size mitochondria, consecutively to decrease in $\Delta \psi$ and depolarisation (Galloway et al. 2012, Lee and Yoon 2014). The fission process is often viewed as division of mitochondria, that facilitate quality control degradation of this organelle (Friedman et al. 2011, Gao et al. 2014, Lackner 2014). Other biological advantages of mitochondrial fission consists in generation of new mitochondria and in their correct distribution according to cells ATP requirement (EscobarHenriques and Anton 2013, Youle and van der Bliek 2012). Inside the cell, mitochondrial fission is initiated at the sites where OMM closely associates with ER/ SR cisternae, and is mediated by dynamin-related protein-1 (DRP1) and fission-1 (FIS1) (Anand et al. 2014, Friedman et al. 2011, Kluge et al. 2013). Within diabetic myocardium, dysfunctional capillary ECs display increased numbers of smaller mitochondria (Figure 2c); this condition is related to cells hyperproliferative phenotype, to augmented expression of FIS1 and DRP1, and to generation of higher ROS levels (Hill 2013, Shenouda et al. 2011). Interestingly, blocking mitochondrial fission (in vitro) impeded high glucose-induced ROS generation and apoptosis ( $\mathrm{Yu}$ et al. 2008). In diabetic CMs, fission of IFM conduct to formation of numerous small size mitochondria, as shown by electron microscopy images (Figure 2d). Perturbation of mitochondrial fission is related to fluctuation of IMM potential, to decreased MFN2/ DRP-1 ratio and to augmented mitophagy, as occurs in heart failure (Galloway et al. 2012, Givvimani et al. 2014).

Mitophagy (elimination of dysfunctional mitochondria by autophagy) consists in engulfment of dysfunctional mitochondria within autophagosomes, followed by fusion of the latter with lysosomes and hydrolysis (Gao et al. 2014). Mitophagy operates as an early cardioprotective response against stressors, implicating the disposal of dysfunctional mitochondria and prevention of their intracellular accumulation (Kobayashi and Liang 2015). However, increased mitophagy may conduct to heart failure, as a result of mitochondria excessive removal and loss of CMs (Kubli and Gustafsson 2012). Mitophagy is triggered by several circumstances: the loss of electrochemical potential, mtDNA mutations, diabetic cardiomyopathy, heart failure, and ischemia/reperfusion (Givvimani et al. 2014, Kobayashi and Liang 2015, Kubli and Gustafsson 2012, Shutt and McBride 2013).

MDV pathway controls also the dysfunctional mitochondria; the vesicles form independently of fission factor DRP1, require PINK1 expression, and target to lysosomes in an autophagy-independent process (McLeland et al. 2014). 
The Molecular Crosstalk Between Mitochondria, Cell Organelles and Cytoplasm

Besides the role of mitochondria as energy power plants of the cells, the recent publications highlight an evolutionary conserved process carried out by these organelles, i.e. the retrograde signaling from mitochondria to the other cellular organelles and to cytoplasm (Pagliarini and Rutter 2013). Apparently, OMM serves as the major signaling platform (Tait and Green 2012). The mitochondrion-to-nucleus communication involves multiple elements: codification of a largest part of mitochondrial proteins by cellular nucleus (including the hem-dependent proteins, such as cytochrome c, catalase and cytochrome oxidase), coordination between mitochondrial signals and nuclear gene expression, and accumulation of nuclear ROS as a result of perinuclear mitochondria clustering (Al-Mehdi et al. 2012, Eynon et al. 2011, Dai et al. 2012, Shimasaki et al. 2013). The above crosstalk may be enabled by perinuclear mitochondria of diabetic CMs (Figure 1c).

Mitochondria have contact sites with ER/ SR, as exemplified here for diabetic CMs (Figure 3a). These contact sites play functional roles, e.g. regulation of lipid synthesis and $\mathrm{Ca}^{2+}$ signaling, control of mitochondriogenesis, and intracellular transport (Rowland and Voeltz 2012). Moreover, the recent reports indicate that contacts function is regulated by ubiquitin, and that ER-mitochondria encounter structure (ERMES) is the spot where initiation of mitochondrial fission process takes place (Eisner et al. 2013, Friedman et al. 2011, Mafalda and Langer 2014). Phospholipids and $\mathrm{Ca}^{2+}$ signals are transported/propagated via ER/SR - mitochondria route, while phosphatidylethanolamine is the main cargo transported from mitochondria to ER/SR (Eisner et al. 2013, Scharwey et al. 2013). Moreover, IMM and OMM are engaged in the intra-mitochondrial transport of lipids (Scharwey et al. 2013).

Mitochondria interact also with lipid droplets, the storage area of triacylglycerols and steryl esters; in heart steatosis, clustering of mitochondria around lipid droplets enable the lipid transfer between these organelles (Figure $3 b)$. 
Figure 3. Electron Microscopy Evidence of Mitochondria Interaction with SR and Lipid Droplets within Diabetic Myocardium. (a) Mitochondria-SR Close Vicinity (*); (b) Mitochondria Clustering around Lipid Droplets (ld), and Adjacent to a myocardial capillary

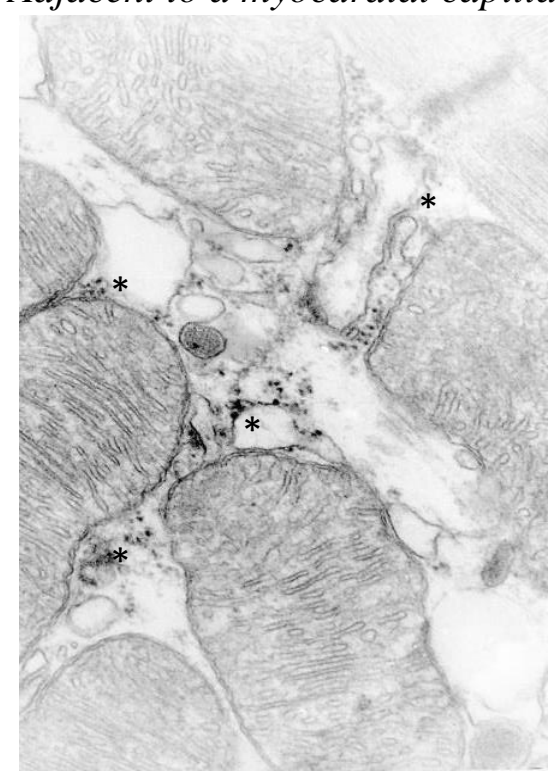

a

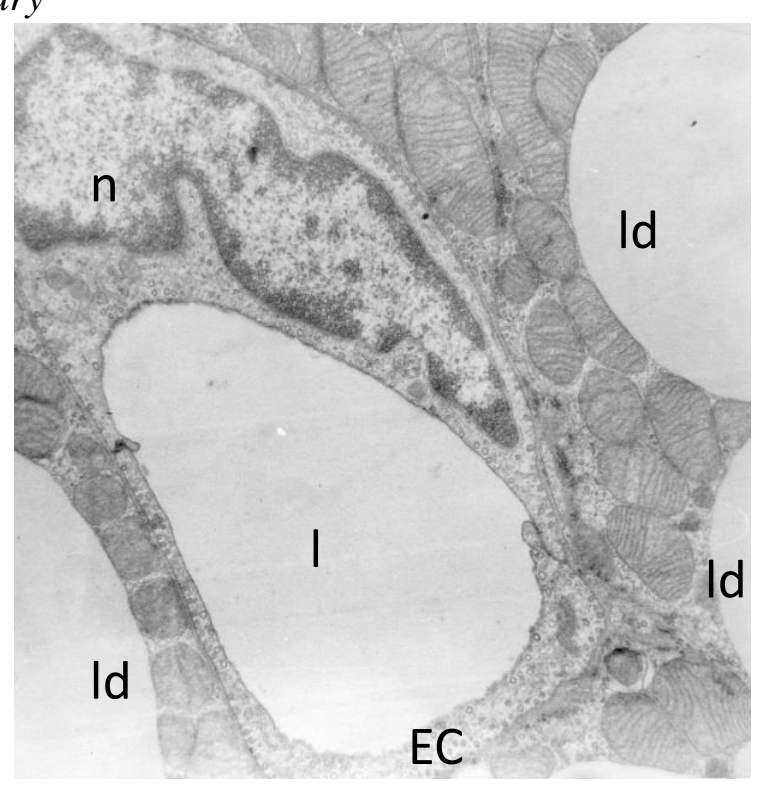

b

Abbreviations: n: nucleus; 1: capillary lumen; EC: endothelial cell. Original magnifications: (a) x 54,600; (b) x 19,200.

Regarding mitochondria - cytoplasm crosstalk, organelles translocation to subcellular sites allows control of local ROS concentrations, of ATP/ADP/ $\mathrm{AMP}$ and $\mathrm{NAD}{ }^{+} / \mathrm{NADH}$ ratios, and of $\mathrm{Ca}^{2+}$ signaling (Al-Mehdi et al. 2012, Picard et al. 2013). When metabolic perturbations occur (such as ER stress characterized by accumulation of unfolded/misfolded proteins beyond the chaperones capacity), mitochondria trigger the unfolded protein response (UPRmt) leading to transcriptional activation of chaperone proteins and proteases that ultimately alleviate ER stress (Papa and Germain 2014, Pellegrino et al. 2013). Furthermore, in conditions of prevalent mitochondrial damages, the organelle releases cytochrome c and activates caspases along with the apoptotic process (Groenewoud and Zwartkruis 2013, Shimasaki et al. 2013). A protective role against apoptosis/necrosis plays activation of mitochondrial AKT1, by suppression of mitochondrial calcium overload, and relief of membrane depolarization (Deng et al. 2014).

\section{Perspectives}

Understanding mechanisms of organelles dialogue in diabetic heart is an ongoing pursuit aimed to be translated into novel appropriate drugs that preserve $\mathrm{CMs}$ viability and alleviate diabetes-induced cardiac damage. A critical evaluation of current literature shows that promising research is focused 
mainly on regulation of mitochondrial turnover, substantiation of dynamics mediators role(s), improvement of quality control efficiency, and of ECs mitochondrial function recovery, identification of metabolomic and lipidomic footprints of metabolic flexibility, and development of pharmaceutical compounds that improve mitochondrial metabolism in humans (Dai et al. 2012, Gao et al. 2014, Kluge et al. 2013, Kubli and Gustafsson 2012, Shutt and McBride 2013). Equally important is elucidation of UPRmt pathway (Pellegrino et al. 2013) and of the links between mitochondrial ROS production and mitochondrial retrograde signaling in the context of cardiovascular aging (Dai et al. 2012).

\section{References}

Al-Mehdi AB, Pastukh VM, Swiger BM, Reed DJ, Patel MR, Bardwell GC, Pastukh VV, Alexeyev MF, Gillespie MN (2012) Perinuclear mitochondrial clustering creates an oxidant-rich nuclear domain required for hypoxia-induced transcription. Science Signaling 5(231): ra47.

Anand R, Wai T, Baker MJ, Kladt N, Schauss AC, Rugarli E, Langer T (2014) The iAAA protease YME1L and OMA1 cleave OPA1 to balance mitochondrial fusion and fission. Journal of Cell Biology 204(6): 919-929.

Baker MJ, Lampe PA, Stojanovski D, Korwitz A, Anand R, Tatsuta T, Langer T (2014) Stress-induced OMA1 activation and autocatalytic turnover regulate OPA1-dependent mitochondrial dynamics. EMBO Journal 33(6): 578-593.

Barazzoni R (2012) Modulating mitochondrial fission to lower diabetic oxidative stress. Diabetes 61(8): 1915-1917.

Baseler WA, Dabkowski ER, Williamson CL, Croston TL, Thapa D, Powell MJ, Razunguzwa TT, Hollander, JM (2011) Proteomic alterations of distinct mitochondrial subpopulations in the type 1 diabetic heart: contribution of protein import dysfunction. American Journal of Physiology-Regulatory, Integrative and Comparative Physiology 300(2): R186-R200.

Bubolz AH, Mendoza SA, Zheng X, Zinkevich NS, Li R, Gutterman DD, Zhang DX (2012) Activation of endothelial TRPV4 channels mediates flow-induced dilation in human coronary arterioles: role of $\mathrm{Ca}^{2+}$ entry and mitochondrial ROS signaling. American Journal of Physiology-Heart and Circulatory Physiology 302(3): H634-H642.

Dai D-F, Rabinovitch PS, Ungvari Z (2012) Mitochondria and cardiovascular aging. Circulation Research 110: 1109-1124.

Davidson SM (2011) A needle in a haystack: focus on "Proteomic alterations of distinct mitochondrial subpopulations in the type 1 diabetic heart". American Journal of Physiology-Regulatory, Integrative and Comparative Physiology 300(2): R183-R185.

Deng W, Leu HB, ChenY, Chen YH, Epperson CM, Juang C, Wang PH (2014) Protein kinase B (PKB/AKT1) formed signaling complexes with mitochondrial proteins and prevented glycolytic energy dysfunction in cultured cardiomyocytes during ischemia-reperfusion injury. Endocrinology 155(5): 1618-1628.

Dominic EA, Ramezani A, Anker SD, Verma M, Mehta N, Rao M (2014) Mitochondrial cytopathies and cardiovascular disease. Heart 100(8): 611-618. 
Eisner V, Csordás G, Hajnóczky G (2013) Interactions between sarco-endoplasmic reticulum and mitochondria in cardiac and skeletal muscle - pivotal roles in $\mathrm{Ca}^{2+}$ and reactive oxygen species signaling. Journal of Cell Science 126(14): 29652978.

Escobar-Henriques M, Anton F (2013) Mechanistic perspective of mitochondrial fusion: tubulation vs. fragmentation. Biochimica et Biophysica Acta 1833(1): $162-175$.

Eynon N, Morán M, Birk R, Lucia A (2011) The champions' mitochondria: is it genetically determined? A review on mitochondrial DNA and elite athletic performance. Physiological Genomics 43(13): 789-798.

Friedman JR, Lackner LL, West M, DiBenedetto JR, Nunnari J, Voeltz GK (2011) ER tubules mark sites of mitochondrial division. Science 334(6054): 358-362.

Galloway CA, Lee H, Nejjar S, Jhun BS, Yu T, Hsu W, Yoon Y (2012)Transgenic control of mitochondrial fission induces mitochondrial uncoupling and relieves diabetic oxidative stress. Diabetes 61(8): 2093-2104.

Galluzzi L, Kepp O, Trojel-Hansen C, Kroemer G (2012) Mitochondrial control of cellular life, stress and death. Circulation Research 111(9): 1198-1207.

Gao AW, Cantó C, Houtkooper RH (2014) Mitochondrial response to nutrient availability and its role in metabolic disease. EMBO Molecular Medicine 6(5): 580-589.

Givvimani S, Pushpakumar S, Veeranki S, Tyagi SC (2014) Dysregulation of Mfn2 and Drp-1 proteins in heart failure. Canadian Journal of Physiology and Pharmacology 92(7): 583-591.

Groenewoud MJ, Zwartkruis FJ (2013) Rheb and mammalian target of rapamycin in mitochondrial homoeostasis Open Biology 3(12): 130-185.

Hill BG (2013) Recent advances in mitochondrial research. Circulation Research 113(12): e107-e110.

Hollander JM, Thapa D, Shepherd DL (2014) Physiological and structural differences in spatially distinct subpopulations of cardiac mitochondria: influence of cardiac pathologies. American Journal of Physiology-Regulatory, Integrative and Comparative Physiology 307(1): H1-H14.

Holmuhamedov EL, Oberlin A, Short K, Terzic A, Jahangir A (2012) Cardiac subsarcolemmal and interfibrillar mitochondria display distinct responsiveness to protection by diazoxide. PLoS One 7(9).

Huang X, Sun L, Ji S, Zhao T, Zhang W, Xu J, Zhang J, Wang Y, Wang X, FranziniArmstrong C, Zheng M, Cheng H (2013) Kissing and nanotunneling mediate intermitochondrial communication in the heart. PNAS 110: 2846-2851.

Ikeda Y, Sciarretta S, Nagarajan N, Rubattu S, Volpe M, Frati G, Sadoshima J (2014) New insights into the role of mitochondrial dynamics and autophagy during oxidative stress and aging in the heart. Oxidative Medicine and Cellular Longevity Article ID 210934.

Jin SM, Youle RJ (2012) PINK1- and Parkin-mediated mitophagy at a glance. Journal Cell Science 125(4): 795-799.

Kluge MA, Fetterman JL, Vita JA (2013) Mitochondria and endothelial function. Circulation Research 112(8): 1171-1188.

Kobayashi S, Liang Q (2015) Autophagy and mitophagy in diabetic cardiomyopathy. Biochimica et Biophysica Acta 1852(2): 252-261.

Kubli DA, Gustafsson AB (2012) Mitochondria and mitophagy: the yin and yang of cell death control. Circulation Research 111(9): 1208-1221.

Lackner LL (2014) Shaping the dynamic mitochondrial network. BMC Biology 12: 35. 
Lee H, Yoon Y (2014) Transient contraction of mitochondria induces depolarization through the inner membrane dynamin OPA1 protein. The Journal of Biological Chemistry 289(17): 11862-11872.

Mafalda E-H, Langer T (2014) Dynamic survey of mitochondria by ubiquitin. EMBO Reports 15(3): 231-243.

Marín-García J (2013) Mitochondria and their role in cardiovascular disease. Springer Science and Business Media (Nov 19): 29-30.

Marzetti E, Csiszar A, Dutta D, Balagopal G, Calvani R, Leeuwenburgh C (2013) Role of mitochondrial dysfunction and altered autophagy in cardiovascular aging and disease: from mechanisms to therapeutics. American Journal of PhysiologyHeart and Circulatory Physiology 305(4): H459-H476.

McBride H, Scorrano L (2013) Mitochondrial dynamics and physiology. Biochimica et Biophysica Acta 1833(1): 148-149.

McLelland G-L, Soubannier V, Chen CX, McBride HM, Fon EA (2014) Parkin and PINK1 function in a vesicular trafficking pathway regulating mitochondrial quality control. The EMBO Journal 33(4): 282-295.

Pagliarini DJ, Rutter J (2013) Hallmarks of a new era in mitochondrial biochemistry. Genes and Development 27(24): 2615-2627.

Palikaras K, Tavernarakis N (2014) Mitochondrial homeostasis: the interplay between mitophagy and mitochondrial biogenesis. Experimental Gerontology 56: 182188.

Papa L, Germain D (2014) SirT3 regulates the mitochondrial Unfolded Protein Response. Molecular and Cellular Biology 34: 699-710.

Papanicolaou KN, Phillippo MM, Walsh K (2012) Mitofusins and the mitochondrial permeability transition: the potential downside of mitochondrial fusion. American Journal of Physiology-Heart and Circulatory Physiology 303(3): H243-H255.

Pellegrino MW, Nargund AM, Haynes CM (2013) Signaling the mitochondrial unfolded protein response. Biochimica et Biophysica Acta 1833(2): 410-416.

Picard M, Shirihai OS, Gentil BJ, Burelle Y (2013) Mitochondrial morphology transitions and functions: implications for retrograde signaling? American Journal of Physiology-Regulatory, Integrative and Comparative Physiology 304(6): R393-R406.

Rowland AA, Voeltz GK (2012) Endoplasmic reticulum-mitochondria contacts: function of the junction. Nature Reviews Molecular Cell Biology 13(10): 607625.

Schaper J, Meiser E, Stämmler G (1985) Ultrastructural morphometric analysis of myocardium from dogs, rats, hamsters, mice, and from human hearts. Circulation Research 56(3): 377-391.

Scharwey M, Tatsuta T, Langer T (2013) Mitochondrial lipid transport at a glance. Journal of Cell Science 126(23): 5317-5323.

Shekar KC, Li L, Dabkowski ER, Xu W, Ribeiro RF Jr, Hecker PA, Recchia FA, Sadygov RG, Willard B, Kasumov T, Stanley WC (2014) Cardiac mitochondrial proteome dynamics with heavy water reveals stable rate of mitochondrial protein synthesis in heart failure despite decline in mitochondrial oxidative capacity. Journal of Molecular and Cell Cardiology 75: 88-97.

Shenouda SM, Widlansky ME, Chen K, Xu G, Holbrook M, Tabit CE, Hamburg NM, Frame AA, Caiano TL, Kluge MA, Duess MA, Levit A, Kim B, Hartman ML, Joseph L, Shirihai OS, Vita JA (2011) Altered mitochondrial dynamics contributes to endothelial dysfunction in diabetes mellitus. Circulation 124(4): 444-453. 
Shimasaki Y, Pan N, Messina LM, Li C, Chen K, Liu L, Cooper MP, Vita JA, Keaney JF Jr (2013) Uncoupling protein 2 impacts endothelial phenotype via p53mediated control of mitochondrial dynamics. Circulation Research 113(7): 891901.

Shlevkov E, Schwarz TL (2014) Have you seen? For Parkin, it's not all or nothing. EMBO Journal 33(4): 277-279.

Shutt TE, McBride HM (2013) Staying cool in difficult times: mitochondrial dynamics, quality control and the stress response. Biochimica et Biophysica Acta 1833(2): 417-424.

Srisakuldee W, Makazan Z, Nickel BE, Zhang F, Thliveris JA, Pasumarthi KB, Kardami E (2014) The FGF-2-triggered protection of cardiac subsarcolemmal mitochondria from calcium overload is mitochondrial connexin 43-dependent. Cardiovascular Research 103(1): 72-80.

Tait SW, Green DR (2012) Mitochondria and cell signalling. Journal of Cell Science 125(4): 807-815.

Wang X, Jian C, Zhang X, Huang Z, Xu J, Hou T, Shang W, Ding Y, Zhang W, Ouyang M, Wang Y, Yang Z, Zheng M, Cheng H (2012) Superoxide flashes: elemental events of mitochondrial ROS signaling in the heart. Journal of Molecular and Cell Cardiology 52(5): 940-948.

Youle RJ, van der Bliek AM (2012) Mitochondrial fission, fusion, and stress. Science 337(6098): 1062-1065.

Yu T, Sheu SS, Robotham JL, Yoon Y (2008) Mitochondrial fission mediates high glucose-induced cell death through elevated production of reactive oxygen species. Cardiovascular Research 79(2): 341-351.

Zhang M, Shah AM (2014) ROS signalling between endothelial cells and cardiac cells. Cardiovascular Research 102(2): 249-257. 
